Article original

\title{
Répertoire et contextes sociaux des cris unitaires du colobe vert (procolobus verus) dans le Parc National de Taï (PNT), Côte d'Ivoire.
}

\author{
Koffi J-C. BenE ${ }^{1,2 *}$, Inza Kone ${ }^{1,2} \&$ Klaus ZuberbuhleR ${ }^{2,3}$ \\ 'Laboratoire de Zoologie et Biologie Animale, UFR Biosciences, Université de Cocody Abidjan, 22 BP 582 Abidjan 22 \\ ${ }^{2}$ Centre Suisse de Recherches Scientifiques, Taï Monkey Project, Abidjan, 01 BP 1303 Abidjan 01. \\ ${ }^{3}$ School of Psychology, University of St Andrews, St Andrews, KY16 9J, Scotland (UK) \\ *Auteur pour les correspondances (E-mail : koffi.bene@csrs.ci ou jeanclaudebene@yahoo.fr) \\ Reçu le 24-04-2006, accepté le 12-11-2006.
}

\begin{abstract}
Résumé
Pour caractériser l'environnement social et écologique du Colobe Vert (Procolobus verus), des observations régulières par tranche de 15 minutes d'animaux focaux dans trois groupes ont été méné. Les observations et les enregistrements de cris occasionnels (Ad libitum) ont permis de répertorier huit (8) types de cris unitaires émis par différentes classes de sexes et d'âges. Ces cris peuvent être regroupés en quatre catégories selon les contextes sociaux dans lesquels ils interviennent : les cris $a, b$ et $c$ en cas de prédation et de trouble (présence de prédateur, cris d'alarme d'autres espèces), les cris $f$ et $g$ en situation de conflit (combat entre individus), les cris b, c et e en contexte de stabilité (calme apparent), et les cris b, c et e pour signaler des rencontres inter-groupes (rencontre avec d'autres groupes de singes). Mots clés : Colobe Vert, vocalisation, cri unitaire, contexte social, communication, Parc National de Taï, Côte d'Ivoire.
\end{abstract}

\begin{abstract}
Repertoire and social contexts of Olive Colobus' (Procolobus verus) unit calls in the Taï National Park (TNP), Ivory Coast.

In order to knod how Olive Colobus monkeys (Procolobus verus) interact with their social and ecological environment, we carried out regular observations by 15 minutes old section of focal animals in three groups. These observations associated with the recordings of occasional calls (Ad libitum) enabled us to index eight (8) types of unit calls emitted by various sex and age classes. These calls can be gathered into four types according to social contexts in which they happened : the calls $a, b$ and $c$ in context of predation and disturbance (presence of predator, alarm calls from other monkey species), the calls $f$ and $g$ in a situation of conflict (fights between individual), the calls $b, c$ and $e$ as a signal of stability (peaceful context), and the calls $b, c$ and $e$ in case of intergroup encounters (encounter with other monkey groups).
\end{abstract}

Key words : Olive Colobus, vocalization, unit call, social context, communication, Taï National Park, Ivory Coast.

\section{Introduction}

L'étude de la communication vocale chez les populations de Primates non-humains dans leurs milieux naturels a connu un essor ces dernières années (Hauser, 1998; Palombit et al., 1999; Rendall \& Cheney, 2000; Fischer et al., 2001; Seyfarth \& Cheney, 2003). Cependant, les espèces vivant dans des habitats comme la savane ont été les plus étudiées par rapport à 
celles des forêts. D'un point de vue évolutionniste, ce déficit constitue un handicap parce que la forêt tropicale est l'un des habitats clés des Primates non-humains et représente une force sélective majeure sur les capacités cognitives des Primates. En effet, la visibilité dans les forêts tropicales étant souvent extrêmement faible, les vocalisations restent le moyen le plus efficient pour la transmission d'informations sociales et écologiques.

De nombreuses études ont mis en évidence la sémanticité des cris émis par les Singes, notamment face à leurs prédateurs. En effet, chaque type de prédateur suscite chez les Singes, l'émission d'un cri d'alarme spécifique (Strushaker, 1967; Seyfarth et al., 1980; Zuberbühler et al., 1997; Hauser, 1998; Zuberbühler, 2000a, 2002). Ainsi, le Cercopithèque Diane (Cercopithecus diana diana) et la Mone de Campbell (C. campbelli) du Parc National de Taï (PNT), en Côte d'Ivoire produisent des cris d'alarme qui indiquent de façon spécifique, la présence de la Panthère (Panthera pardus) ou celle de l'Aigle Couronné (Stephanoeatus coronatus), deux de leurs principaux prédateurs (Zuberbühler, 2000a, 2001). En savane, les études menées sur les Babouins de Chacma (Papio cynocephalus ursinus) ont montré que des individus utilisent diverses vocalisations bien précises dans différents contextes sociaux (Cheney et al., 1995; Cheney \& Seyfarth, 1997; Rendall \& Cheney, 2000; Kitchen et al., 2003). Les Primates de forêt produisent de tels types de vocalisations, qui interviennent dans le contrôle de la proximité spatiale des membres du groupe (Gautier \& Gautier, 1977). Ces cris donnent aux membres du groupe, des informations sur des événements récurrents dans leur environnement (Uster \& Zuberbühler, 2001). De plus, les études récentes sur la Mone de Campbell en captivité suggèrent que ces cris permettent de véhiculer des messages comportant des informations sur l'état social des individus (Lemasson et al., 2003).

Dans la forêt du PNT vivent sympatriquement trois espèces de colobiné : le Colobe Bai d'Afrique de l'Ouest (Procolobus [Piliocolobus] badius), le Colobe Blanc-et-Noir d'Afrique de l'Ouest (Colobus polykomos) et le Colobe Vert (Procolobus verus). Le Colobe Blanc-et-Noir est le plus gros et le Colobe Vert, le plus petit des trois. Si le répertoire vocal du Colobe Blanc-et-
Noir ainsi que celui du Colobe Bai ont été établis, il n'en est pas le cas du Colobe Vert. En effet, les Colobes Vert ont été étudiés dans leurs milieux naturels sur l'lle de Tiwai (Sierra Leone) et dans le PNT, mais leur comportement vocal n'a jamais été systématiquement décrit (Booth, 1957; GalatLuong, 1983; Oates, 1984, 1988 ; Whitsides, 1989 ; Oates \& Whitesides, 1990; McGraw, 1996, 1998 ; Höner et al., 1997; Davies et al., 1999; Korstjens, 2001). Ces études ont montré que les individus forment des associations presque permanentes avec un groupe particulier de Cercopithèque Diane, même dans les habitats où la pression de prédation est relativement faible (Oates, 1984; Bshary \& Noë, 1997a). En plus, des mâles adultes ainsi que des femelles ont été observés changeant de groupe. Ceci explique l'existence chez le Colobe Vert, d'un système social remarquablement fluide selon plusieurs modèles, avec des groupes d'un mâle et de plusieurs femelles et des groupes de plusieurs mâles et plusieurs femelles (Korstjens, 2001).

Des observations pilotes ont suggéré que le Colobe Vert possède un répertoire vocal relativement peu varié, mais que les individus sont capables de combiner plusieurs de leurs cris en des séquences complexes, comme cela a été récemment rapporté chez le Cercopithèque de Campbell (Zuberbühler, 2002).

Cette étude se propose de répondre à la question de savoir quels sont les différents types de cris unitaires du Colobe Vert du PNT et quels sont les contextes sociaux d'émission de ces cris ?

\section{Matériel et méthodes}

\subsection{Site d'étude}

Le Parc National de Taï a servi de cadre à notre étude. Ce parc est caractérisé par la présence d'une végétation dense humide de type "sempervirente ou forêt ombrophile ". Le PNT est situé au sud-ouest de la Côte d'lvoire entre les latitudes 5'08 et 6'07 nord et les longitudes 0 '47 et 7'25 ouest. Le site d'étude se situe à environ $1 \mathrm{~km}$ à l'est de la station du Centre de Recherches en Ecologie (CRE) située à une dizaine de kilomètres de la frontière ouest du parc, à la hauteur du village de Paulé Oula. Cette forêt abrite environ 140 espèces de Mammifères dont 12 espèces de Primates (Rieizebos E. P. et al., 1994; Hoppe-Dominik, 1995). 


\subsection{Animaux et groupes sociaux}

Les observations ont porté sur 20 individus repartis en trois groupes (groupe 1 , groupe 2 et groupe 3 ). Ces 20 individus comprennent trois mâles adultes, neuf femelles adultes, deux juvéniles et six petits. Les adultes représentent les individus dont les caractères sexuels secondaires sont développés et bien visibles. Les juvéniles sont ceux dont les caractères sexuels secondaires ne sont pas visibles et les petits, les individus qui continuent de taiter (Hsu \& Lin, 2001).

\subsection{Collecte des données}

Trois techniques ont été utilisées pour la collecte des données entre les mois de juin 2001 et février 2002: l'observation d'animal focal, des observations Ad libitum et l'enregistrement de cris.

\subsubsection{Observation d'animal focal}

Tous les individus des trois groupes sont bien identifiés. Chaque groupe est suivi pendant deux jours. Au cours d'une journée, chaque individu est suivi sur une période de $15 \mathrm{~min}$, et ce, plusieurs fois de manière alternative. Les observations commencent généralement à 07 heures pour prendre fin à 17 heures 30 minutes. Le premier individu à observer est choisi au hasard, puis on marque une pause de 15 minutes entre deux individus cibles. Le choix de l'individu suivant se fait de manière à ne pas suivre un même individu deux fois de suite. On récolte alors des informations sur le comportement de l'individu focal et ceux liés au milieu suivant les paramètres contenus dans le tableau 1.

\subsubsection{Observation Ad libitum}

En dehors de la période de collecte des données précédentes (pendant la période de la pause de 15 minutes), tous les cris émis par le Colobe Vert sont enregistrés en utilisant les variables du tableau 1. Ceci a permis, en addition avec les cris des observations focales, de recenser tous les cris émis par le groupe au cours de la journée.

\subsubsection{L'enregistrement des cris}

II s'est réalisé à l'aide d'un microphone Sennheiser connecté à un enregistreur de type « walkman professional ».

Tableau 1 : Description des différents paramètres environnementaux et sociaux

\begin{tabular}{|c|c|c|}
\hline Variables & Modalités & Description \\
\hline Individu & $\begin{array}{l}\text { Mâle adulte } \\
\text { Femelle adulte } \\
\text { Juvénile }\end{array}$ & $\begin{array}{l}\text { Mâle ayant atteint la maturité sexuelle } \\
\text { Femelle ayant atteint la maturité sexuelle } \\
\text { Jeune mais n'ayant pas atteint la maturité sexuelle }\end{array}$ \\
\hline Cris & $\begin{array}{l}\text { Unitaires } \\
\text { Combinés }\end{array}$ & $\begin{array}{l}\text { Type de cri émis seul } \\
\text { Types de cris en combinaison }\end{array}$ \\
\hline Eclairement général & $\begin{array}{l}\text { Sombre } \\
\text { Moyen } \\
\text { Eclairé }\end{array}$ & $\begin{array}{l}\text { Absence d'ombre au sol, ciel couvert } \\
\text { Ciel modérément éclairé } \\
\text { Ciel très éclairé }\end{array}$ \\
\hline Densité de la végétation & $\begin{array}{l}\text { Dense } \\
\text { Moyenne } \\
\text { Ouverte }\end{array}$ & $\begin{array}{l}\text { Touffue, canopée invisible } \\
\text { Modérée ou peu touffue } \\
\text { Cimes de certains arbres visibles }\end{array}$ \\
\hline $\begin{array}{l}\text { Localisation dans le } \\
\text { domaine vital }\end{array}$ & $\begin{array}{l}\text { Bordure } \\
\text { Centre }\end{array}$ & $\begin{array}{l}\text { Environ } 100 \mathrm{~m} \text { de la limite du territoire } \\
\text { A l'intérieur du territoire, jusqu'à } 100 \mathrm{~m} \text { avant la limite }\end{array}$ \\
\hline Groupes voisins & $\begin{array}{l}\text { Mixte } \\
\text { Proche } \\
\text { Lointain }\end{array}$ & $\begin{array}{l}\text { Les groupes sont à } 0 \mathrm{~m} \\
\text { Groupes séparés d'environ } 50 \mathrm{~m} \\
\text { Groupes séparés d'au moins } 100 \mathrm{~m}\end{array}$ \\
\hline $\begin{array}{l}\text { Associations } \\
\text { polyspécifiques }\end{array}$ & $\begin{array}{l}\text { Mixte } \\
\text { Proche } \\
\text { Lointain }\end{array}$ & $\begin{array}{l}\text { Les groupes sont à } 0 \mathrm{~m} \\
\text { Groupes séparés d'environ } 50 \mathrm{~m} \\
\text { Groupes séparés d'au moins } 100 \mathrm{~m}\end{array}$ \\
\hline $\begin{array}{l}\text { Comportements } \\
\text { principaux }\end{array}$ & $\begin{array}{l}\text { Déplacement } \\
\text { Jeu } \\
\text { Fourragement } \\
\text { Station assise } \\
\text { Epouillage } \\
\text { Sommeil }\end{array}$ & $\begin{array}{l}\text { Action de se déplacer, locomotion } \\
\text { Action de jouer seul ou avec d'autres individus } \\
\text { Action de se nourrir } \\
\text { Etre assis } \\
\text { Action de se gratter, de gratter ou d'être gratté } \\
\text { Action de dormir }\end{array}$ \\
\hline
\end{tabular}




\subsection{Analyse des données}

L'analyse des cris enregistrés en forêt s'est faite par l'établissement de sonagrammes au moyen des logiciels CoolEdith 2000 et Praat 3.9.31. Ces logiciels nous ont permis de mesurer la durée et la fréquence de chaque type de cri. Le logiciel Adobe Photoshop 6.0 nous a permis d'améliorer les contrastes et les couleurs des sonagrammes. Pour relier les différents types de cris du colobe vert avec les différents contextes d'émission de ces cris, on a procédé à une Analyse en Composantes Principales avec le logiciel XLSTAT. Le test de $\mathrm{Chi}^{2}$ nous a permis de comparer les données avec une différence significative au seuil de $5 \%$.

\section{Résultats}

\subsection{Répertoire des cris unitaires du Colobe Vert}

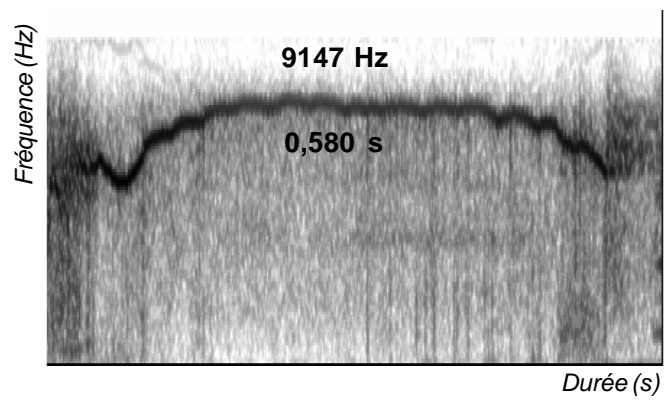

a : Spectrogramme représentant un cri a (zih) émis par un mâle adulte
Le répertoire vocal du Colobe Vert est constitué de huit (8) types de cris unitaires identifiés sous forme de lettres a, b, c, d, e, f, g, h. Ces cris sont émis par des individus de sexes différents et dans divers contextes sociaux regroupés en quatre grands groupes : prédation, conflit, stabilité, rencontre intergroupe.

Trois de ces cris sont fréquemment émis (Fig. 1), ce sont : le cri «zih » ou a (Fig. 1a), le cri « hoo » ou b (Fig. 1b), le cri « zuk » ou c (Fig. 1c). Au total 733 cris unitaires ont été enregistrés dans différents contextes sociaux. Nous avons une dominance nette du cri b $(65,1 \%)$, suivi du cri a $(15,3 \%)$, du cri e $(11,9 \%)$, puis du cris c $(3,5 \%)$. La différence entre ces fréquences de cris est hautement significative $\left(\chi^{2}=196,624 ; \mathrm{df}=24\right.$ et $p<0,001)$. Les autres cris $(d, f, g$ et $h)$ sont en très faibles proportions. Hormis le cri b qui est émis également par les juvéniles, tous ces trois cris sont émis uniquement par les adultes des

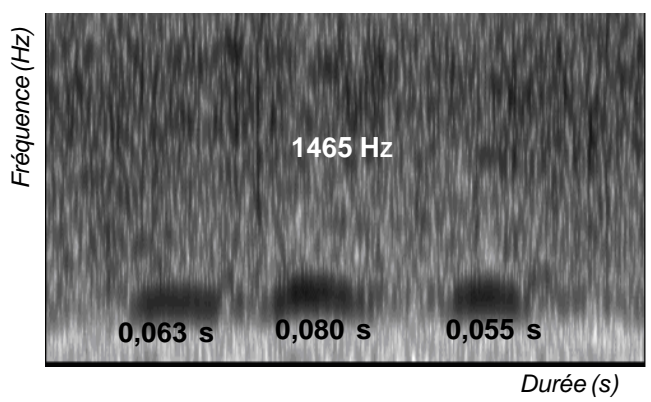

b : Spectrogramme représentant trois (3) cris b (hoo) émis par un mâle adulte

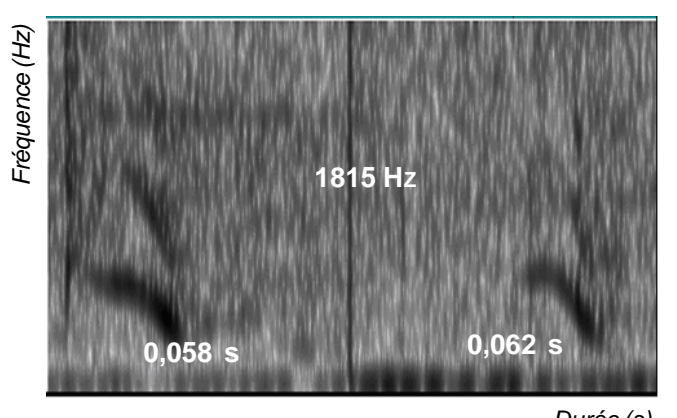

Durée (s)

c : Spectrogramme représentant deux (2) cris c (zuk) émis par un mâle adulte

Figure 1 : Spectrogrammes représentant des cris de mâle adulte de Colobe Vert. 
deux sexes.

\subsection{Contextes sociaux des cris unitaires du Colobe Vert}

L'émission des trois cris commun est suscitée par des facteurs comme la présence de prédateurs, l'émission de cris d'alarme par d'autres Singes, la chute de branches ou d'arbres, ou les rencontres d'autres groupes. En plus, les cris «hoo» et «zuk» sont aussi émis durant les périodes de stabilité, en réponse à des cris de contact du Cercopithèque Diane, ou en réponse à des cris (hoo, zuk, zz, thio) d'autres Colobe Vert. Quant aux autres cris, ils sont émis dans des circonstances moins fréquentes mais relativement bien précises par les femelles et les juvéniles. Ce qui donne des indications claires sur les fonctions de ces cris (Fig. 2).

Le cri «tr » ou cri d (Fig. 2a) est produit par les juvéniles en conjonction avec des gestes de menace, souvent contre l'observateur. En général, l'individu descend au sol, court pour s'arrêter juste à quelques mètres de l'observateur en émettant ce cri.

Le cri « zz » ou cri e (Fig. 2b) est produit par les petits et sert à rétablir le contact avec leurs mères. Ils peuvent entraîner une réponse vocale d'autres individus du groupe. Le cri «ZZ» est aussi produit par les juvéniles au cours des rencontres intergroupes et quelques fois lors des progressions du groupe.

Le cri « whi » ou cri f (Fig. 2c) est produit par les femelles adultes et les juvéniles lorsqu'ils sont agressés par un conspécifique ou par un congénère. Ce cri est émis pendant un mouvement de fuite.

Le cri « ha » ou cri g par contre, (Fig. 2d), émis à la fois par les femelles adultes et par les juvéniles, précède les attaques de l'individu qui l'émet. Ces attaques peuvent être dirigées aussi bien contre des individus de la même espèce que contre des individus d'autres espèces. L'émission de ce cri s'accompagne d'ouvertures de la bouche, signes de menace. Les mâles adultes ne semblent pas produire ce type de cris mais combinent les gestes de menace avec un son non vocal, probablement issu de grincements de dents.

Le cri «thio » ou cri h (Fig. 2e) est émis lorsque l'individu est surpris par un événement, par exemple, lorsqu'il découvre soudainement qu'il est très proche de l'observateur.

L'Analyse en Composantes Principales (ACP), confirme la corrélation entre les cris unitaires et des contextes sociaux précis. En effet, la projection des cris et des contextes sociaux dans le plan 1-2 de l'ACP (Fig. 3), a permis de distinguer quatre groupes de cris reliés à différents contextes sociaux. Ainsi, on distingue le cri f dans les contextes de conflit uniquement, le cri b dans les contextes de stabilité, d'alarme et de vent. Le cri $c$ est émis pendant la chute de branche, les rencontres intergroupes et des bruits d'animaux. Les cris $\mathrm{g}, \mathrm{h}, \mathrm{a}$, d et e sont liés aux contextes de conflit, de chute accidentelle d'un petit, de présence d'observateur très proche et pour le rétablissement du contact entre un petit et sa mère. 


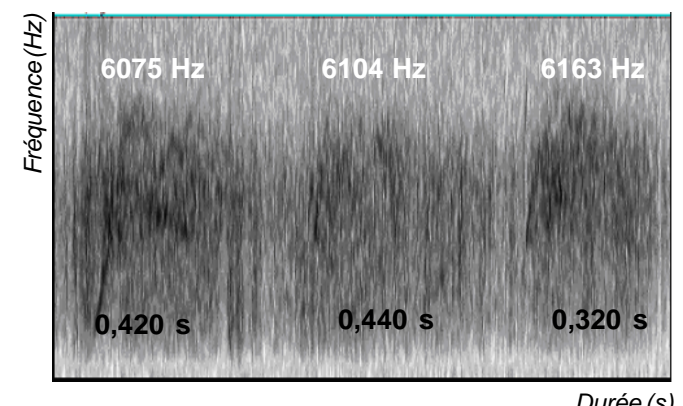

a : Spectrogramme représentant trois (3) cris d (tr) émis par un juvénile.

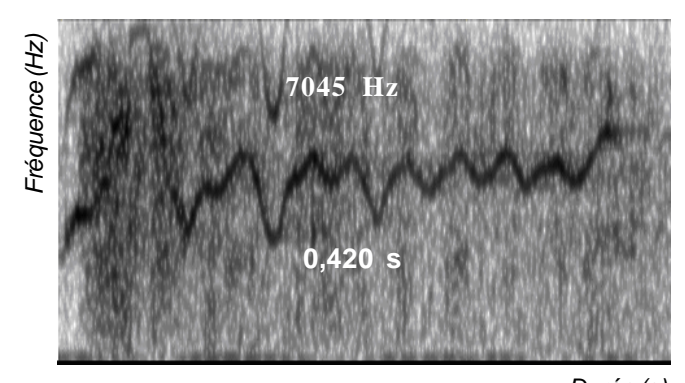

c : Spectrogramme représentant un crif (whi) émis par une femelle adulte.

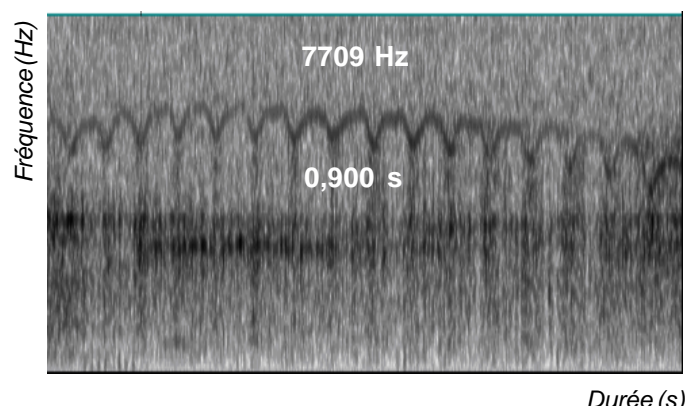

b : Spectrogramme représentant un cri e (zz) émis par un juvénile.

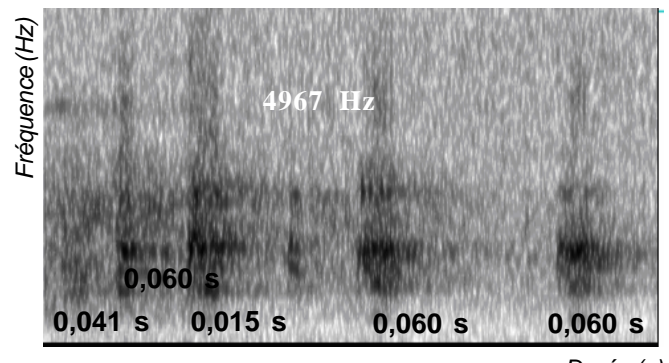

Durée (s)

d : Spectrogramme représentant une série de cris $g$ (ha) émis par un juvénile.

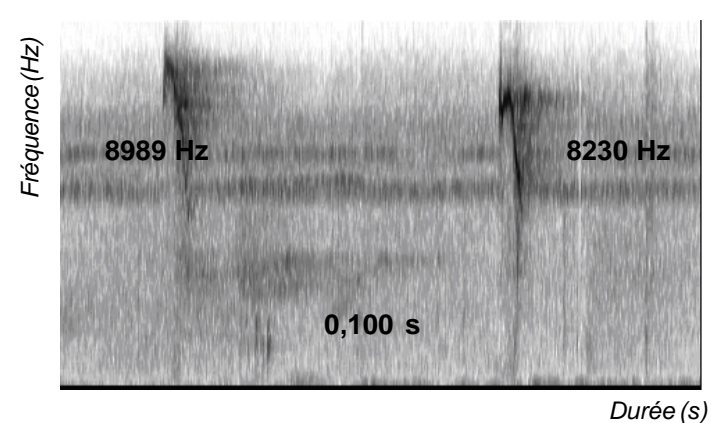

e : Spectrogramme représentant deux (2) cris $\mathrm{h}$ (thio) émis par un juvénile.

Figure 2 : Spectrogrammes représentant les cris de juvénile et de femelle adulte de Colobe Vert 


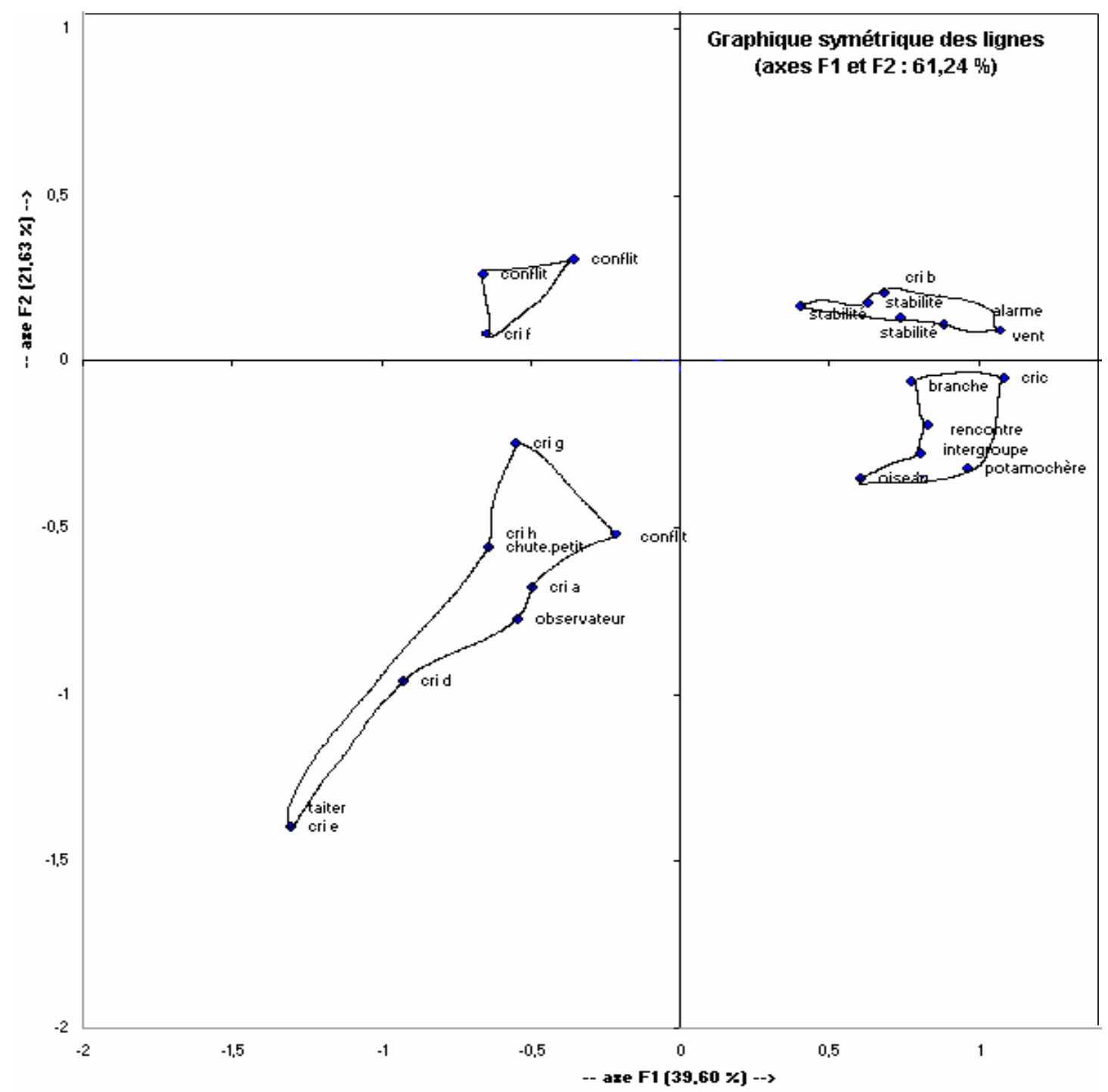

Figure 3 : Analyse en Composantes Principales (ACP) montrant les relations entre les différents types de cris unitaires et les contextes sociaux d'émission des cris. 


\section{Discussion}

Les animaux qui communiquent beaucoup au moyen de la vocalisation sont ceux qui vivent dans des milieux où les autres moyens de communication sont difficilement utilisables. Chez les Singes en plus, l'importance de la communication est liée à la vie en groupe. Cette communication régule des fonctions sociales et les fonctions de reproduction (Gautier \& Gautier, 1977). Les cris émis par le Colobe Vert, peuvent être regroupés en quatre catégories.

\subsection{Cris $a, b$ et $c$ émis en contexte de prédation et de trouble}

Si l'on considère la pression de prédation qui s'exerce sur les Singes de la forêt de Taï, l'on s'attend à voir ces animaux développer un réel système d'alarme (Zuberbühler \& Holenweg, 1991; Zuberbühler et al., 1997). Chaque prédateur ayant un style différent de chasse, suscite une réponse comportementale différente de ces Singes. Les trois cris les plus communs chez le Colobe Vert (zih, hoo, et zuk), ont été produits dans plusieurs contextes, rendant leur analyse fonctionnelle peu précise. Tous ces trois cris sont émis en réponse à des perturbations telles que la présence de prédateurs, l'émission de cris d'alarme par d'autres Singes, la chute d'arbres ou de branches. Ce qui suggère que ces cris fonctionnent comme des cris d'alarme classiques (Zuberbühler, 1999; Zuberbühler \& Jenny, 1999 ; Zuberbühler et al., 1999). Ainsi, le Colobe Vert du PNT produit ces trois types de cris face à la Panthère et à l'Aigle Couronné, mais très souvent en les intégrant dans des combinaisons de cris plus complexes. En revanche, en cas d'attaques des Chimpanzés, le Colobe Vert reste plutôt silencieux et semble, dans ces circonstances, se contenter de réagir au cri d'alarme de la femelle du Cercopithèque Diane, notamment en se cachant ou en fuyant tout en utilisant les strates supérieures dont les branches ne peuvent pas supporter le poids de ce prédateur (Boesch, 1990). Dans ces circonstances, ces Singes restent calmes (Boesch, 1990; Bshary \& Noë, 1997a, b; Noë \& Bshary, 1997) jusqu'à une ou deux heures après. Lorsque le Colobe Vert découvre l'observateur trop près de lui, seuls des cris unitaires sont émis. II s'agit du cri «tr» et «thio» qui font penser au « nyow » émis par le Singe Vert quand il découvre soudainement un petit prédateur (Strushaker, 1967).

\subsection{Cris $b$, $c$ et e émis en Contexte de stabilité}

La limitation de la visibilité en forêt tropicale comme celle de Taï rend les informations vocales plus efficientes que les informations visuelles (Boesch, 1990; Teixidor \& Byrne, 1997). La discrimination vocale devient alors, le moyen le plus efficient pour celui qui écoute de savoir si l'individu émetteur est familier ou pas (Boinski, 1993; Teixidor \& Byrne, 1997). Ainsi, pendant les contextes sans agressivité et paisibles, le Colobe Vert du PNT émet les cris hoo et zuc (cris b et c) en réponse au cri de contact du Cercopithèque Diane, ou en réponse au cri d'un congénère (cris $b, c$ ou e). Ces cris très fréquemment émis qui interviennent dans plusieurs contextes sont des cris de contact et de maintien de la cohésion sociale (Marler, 1970; Gautier \& Gautier, 1977; Snowdon et al., 1983; Palombit, 1992; Uster \& Zuberbühler, 2001). Ceci suggère que ces cris sont produits dans des contextes plus larges que dans une situation de danger.

\subsection{Cris b, c, e émis en contexte de rencontre inter-groupe}

Chacun des groupes étudiés possède son domaine vital qu'il parcourt tous les jours. Au cours de ces tournées, il arrive que des groupes se retrouvent en même temps à une frontière commune. Au cours de ces événements, il n'y a eu aucun affrontement entre les Colobes Vert comme c'est souvent le cas chez le Cercopithèque Diane. Seuls des cris ont été émis. Les cris unitaires émis sont des répétions du cri b ou du cri c qui, le plus souvent, sont des réponses des adultes au cri e des juvéniles. En plus de ces cris unitaires, plusieurs cris combinés sont aussi émis dans les mêmes circonstances. Ces cris de rencontre intergroupe servent à informer les groupes voisins de la présence d'un autre groupe. Ils fonctionnent donc comme le « intergroup grunt » du mâle et le « long aar » des femelles et juvéniles du Singe Vert (Cercopithecus aethiops) (Strushaker, 1967), le « roar » du Colobe Blanc et Noir (Marler, 1972) et le «long cri » du Cercopithèque Diane (Zuberbühler et al., 1997). 


\subsection{Cris $f$ et $g$ émis en contexte de conflit}

Malgré l'entente relative qui existe entre le Colobe Vert et le Cercopithèque Diane, il arrive quelquefois des conflits entre des individus de ces deux espèces de Singes. Ces conflits, ainsi que ceux qui opposent certains membres du groupe de Colobe Vert, entraînent l'émission de deux types de cris ( $f$ (whi) et $g(h a)$ ). Le cri « ha » est un cri de menace qui précède les attaques du Colobe Vert comme c'est le cas du « huh huh »du Singe Rouge (Erythrocebus patas) (Estes, 1991). Cependant, quand le Colobe Vert est attaqué, c'est le «whi » qu'il émet en fuyant. Ce dernier cri fonctionne ainsi comme les cris de soumission « chutter », « woof-woof », « wa », et « woof-wa » du Singe Vert (Strushaker, 1967).

\section{Conclusion}

D'une manière générale, les animaux qui communiquent le plus par voie sonore sont ceux qui vivent dans des milieux où les autres voies de communication sont difficilement utilisables comme c'est le cas des forêts tropicales denses (Gautier \& Gautier, 1977). Notre étude révèle plutôt que le Colobe Vert du PNT, forêt tropicale dense, est une espèce relativement silencieuse. Ce Singe a un répertoire vocal pauvre qu'il utilise rarement. Au nombre de ces cris, huit sont unitaires c'est-à-dire qu'ils peuvent être émis seuls. Ce sont le zih (cri a), le hoo (cri b), le zuk (cric), le tr (cri d), le zz (cri e), le ha (cri f), le whi (cri g) et le thio (cri h). De ceux-ci, cinq interviennent dans des contextes sociaux bien spécifiques comme lors des conflits, avec les cris $d$, $f$ et $g$. En période de stabilité et lors des rencontres intergroupes, c'est le juvénile qui émet souvent le cri e. Par contre, trois cris ( $a$, b et $c)$, interviennent dans différents contextes à la fois. Leur occurrence dans divers contextes qualifiés de troubles dans le groupe permet de dire qu'ils agissent comme des cris d'alarme classiques même si deux d'entre eux (cris c et b) interviennent aussi dans des contextes de stabilité.

\section{Remerciements}

Les auteurs expriment leurs remerciements à l'endroit de la Direction de la Recherche pour avoir autorisé la réalisation de cette étude dans le Parc National de Taï. Le PACPNT est également remercié pour avoir autorisé l'accès au PNT. Ils sont reconnaissants au Centre Suisse de Recherches Scientifiques en Côted'Ivoire (CSRS) pour son appui financier et logistique lors de la réalisation de ces travaux. Ils n'oublient pas les responsables, les étudiants et les assistants du TMP (Taï Monkey Project) dont les conseils et appuis ont été d'un apport considérable pour la réalisation de ces travaux.

\section{Références citées}

Boesch C., 1990. First hunter of the forest. New Scientist, 19 mai, 38-41.

Boinski S., 1993. Vocal coordination of troop movement among white-faced capuchin monkeys (Cebus capucinus). Am. J. Primatol. 30: 85-100.

Booth A.H., 1957. Observations of the natural history of the Olive colobus monkey, Procolobus verus (van Beneden). Proc. Zool. Soc. Lond. 129: 421-430.

Bshary R., \& Noë R., 1997a. Red colobus and Diana monkeys provide mutual protection against predators. Anim. Behav. 54: 1461-1474.

Bshary R., \& Noë R., 1997b. Anti-predation behaviour of red colobus monkeys in the presence of chimpanzees. Behav. Ecol. Sociobiol. 41: 321333.

Cheney D.L., \& Seyfarth R.M., 1997. Reconciliatory grunts by dominant female baboons influence victims' behaviour. Anim. Behav. 54: 409-418.

Cheney D.L., Seyfarth R.M., \& Silk J.B., 1995. The role of grunts in reconciling opponents and facilitating interactions among adult female baboons. Anim. Behav. 50: 249-257.

Davies A.G., Oates J.F., \& Dasilva G.L., 1999. Patterns of frugivory in three West African Colobine monkeys. Int. J. Primatol. 20: 327-357.

Estes, R.D., 1991. The Behavior Guide to African Mammals Including Hoofed Mammals, Carnivores, Primates. University of California Press: Berkeley, California, 611 pp.

Fischer J., Metz M., Cheney D.L., \& Seyfarth R.M., 2001. Baboon responses to graded bark variants. . Anim. Behav. 61: 925-931.

Galat-Luong A., 1983. Socio-écologie de trois colobes sympatriques, Colobus badius, $C$. polykomos et $C$. verus du Parc National de Taï. Université Pierre et Marie Curie Paris VI. 
Gautier J-P, Gautier-Hion A., 1977. Communication in Old World Monkeys. In How animal communicates (Sebeok TE, ed), p: 890-964. Bloomington, Indiana University Press.

Hauser M.D., 1998. Functional referents and acoustic similarity: field playback experiments with rhesus monkeys. Anim. Behav. 55: 1647-1658.

Höner O.P., Lorenz L., \& Noë R. 1997. Dyadic associations of red colobus and Diana monkey groups in Taï National Park, Ivory coast. Primates 38: 281-291.

Hoppe-Dominik, B. (1995). L'état actuel des effectifs des grands mammifères dans l'ensemble du Parc National de Taï. GmbH-GTZ, DPN, Abidjan, 66pp.

Hsu, M.J. \& Lin, J-F., 2001. Troop size and structure in free-ranging Formosan Macaques (Macaca cyclotis) at Mt. Longevity, Taiwan. Zool. Stud. 40 (1): 49-60.

Kitchen D.M., Seyfarth R.M., Fischer J., \& Cheney D.L., 2003. Loud calls as indicators of dominance in male baboons (Papio cynocephalus ursinus). Behav. Ecol. Sociobiol. 53: 374-384.

Korstjens A.H., 2001. The mob, the secret sorority, and the phantoms: an analysis of the socioecological strategies of the three colobines of Taï. PhD Thesis, Utrecht University, 174 pp.

Lemasson A., Gautier J-P., \& Hausberger M., 2003. Vocal similarities and social bonds in Campbell's monkey (Cercopithecus campbelli). C. R. Bilogies 326 : 1185-1193.

Marler P., 1970. Vocalization of East African monkeys. I. Red colobus. Folia Primatol. 13: 81-91.

Marler P., 1972. Vocalizations of East African monkeys, II: Black and white colobus. Behaviour 42: 175-197.

McGraw W.S., 1996. Cercopithecid locomotion, support use, and support availability in the Tai Forest, Ivory Coast. Am. J. Phys. Anthropol. 100: 507-522.

McGraw W.S., 1998. Comparative locomotion and habitat use of six monkeys in the Tai Forest, Ivory Coast. Am .J. Phys. Anthropol. 105: 493-510.

Noë R. \& Bshary R., 1997. The formation of red colobus-diana monkey associations under predation pressure from chimpanzees. Proc. $R$. Soc. Lond. B. 264: 253-259.
Oates J.F., 1984. Association between olive colobus monkey, Procolobus verus, and Cercopithecus species in Sierra Leone. Int. J. of Primatol. 5: 370pp.

Oates J.F., 1988. The diet of the olive colobus monkey, Procolobus verus, in Sierra Leone. Int. J. Primatol. 9: 457-478.

Oates J.F. \& Wheiitesides G.H., 1990. Association between olive colobus (Procolobus verus), Diana guenons (Cercopithecus diana), and other forest monkeys in Sierra Leone. Am. J. Primatol. 21: 129-146.

Palombit, R. A., 1992. A preliminary study of vocal communication in wild long-tailed macaques: II. Potential of calls to regulate intragroup spacing. Int. J. Primatol. 13(2): 183-207.

Palombit R.A., Cheney D.L., \& Seyfarth R.M., 1999. Male grunts as mediators of social interaction with females in wild Chacma baboons (Papio cynocephalus ursinus). Behaviour 136: 221-242.

Rendall D. \& Cheney D.L., 2000. Proximate factors mediating contact calls in adult female baboon (Papio cynocephalus ursinus) and their infants. J. Comp. Psych. 114 (1): 36-46.

Rieizebos E.P., Vooren A. P., \& Guillaumet J. L., 1994. Le Parc National de Taï, Côte d'Ivoire. I) Synthèse des connaissances. II) Bibliographie. (Ed. Sloot, P. H. M. and Hazeu, G. W.) serie Tropenbos $n^{\circ}$ 8, 323pp., Backhuys Publisher. Leiden, Pays-Bas.

Seyfarth R.M., \& Cheney D.L., 2003. Signalers and receivers in animal communication. Annu. Rev. Psychol. 54: 145-173.

Seyfarth R.M., Cheney D.L., \& Marler P., 1980. Vervet monkey alarm call: semantic communication in a free-ranging primate. Anim. Behav. 28: 1070-1094.

Snowdon C.T., Cleveland J., \& Fench J.A., 1983. Responses to context and individual-specific cues in cotton-top-tamarin long calls. Anim. Behav. 31: 92-101.

Strushaker T.T., 1967. Auditory communication among vervet monkeys (Cercopithecus aethiops). University of Chicago Press, Chicago, p: 281-324.

Teixidor P., \& Byrne R.W., 1997. Can spider monkey's (Ateles geoffroyi) discriminate vocalizations of familiar individuals and stranger. Folia Primatol. 68: 254-264. 
Uster D., \& Zuberbühler K., 2001. The functional significance of Diana monkey Clear calls. Behaviour 138: 741-756.

Whitesides G.H., 1989. Interspecific associations of Diana monkeys, Cercopithecus diana, in Sierra Leone, West Africa: Biological significance or chance? Anim. Behav. 37: 760-776.

Zuberbühler K., 1999. Interspecific semantic communication in two forest primates. Proc. $R$. Soc. Lond. B. 267: 713-718.

Zuberbühler K., 2000a. Causal knowledge of predators behaviour in wild Diana monkeys. Anim. Behav. 59: 209-220.

Zuberbühler K., 2000b. Referential labelling in Diana monkeys. Anim. Behav. 59 (5): 917-927.

Zuberbühler K., 2001. Predator-specific alarm calls in Campbell's guenons. Behav. Ecol. Sociobiol. 50: 414-422.
Zuberbühler K., 2002. A syntatic rule in forest monkey communication. Anim. Behav. 63 : 293-299.

Zuberbühler K., Cheney D.L, \& Seyfarth R.M., 1999. Conceptual semantics in nonhuman primate. J. Compar. Psychol. 113: 33-42.

Zuberbühler K., \& Holenweg K., 1991. Projet Colobus - Recherche sur la communication acoustique chez les cercopithecinae vivant dans la forêt du Parc National de Taï - Association entre des groupes de Colobus badius et Cercopithecus diana au Parc national de Taï. Rapports annuel, p: 145-149.

Zuberbühler K., \& Jenny D.B., 1999. The predator deterrence function of primate alarm calls. Ethology, 105: 477-490.

Zuberbühler K., Seyfarth R.M., \& Noë R., 1997. Diana monkey loud calls: messages for conspecifics and predators. Anim. Behav. 53: 589-604. 